\title{
Comparison of Endurance, Agility, and Strength in Elite Hockey and Soccer 9 Year-Old Players
}

\author{
${ }^{1}$ Eryk Przysucha*, ${ }^{1}$ Carlos Zerpa, ${ }^{1}$ Christopher Piek \\ ${ }^{1}$ School of Kinesiology, Lakehead University, Thunder Bay, Ontario, Canada.
}

Submitted 01 September 2018; Accepted in final form 01 January 2019.

\begin{abstract}
Background. Regardless of the age, elite athletes exhibit various motor capabilities (e.g., speed; endurance; strength) that are specific to that particular sport. Objectives. The purpose of this study was to compare different aspects of physical fitness (agility, strength, endurance) in thirty, 9 year old elite soccer and hockey players. Methods. Participants completed a 90 minute familiarization session, and returned at the later date to perform tests examining their agility (T-test), endurance (20mSRT), and strength (long jump; v-ups; push-ups; sit-ups). The tests were administered by an expert trainer at the same location. At the onset no inter-group differences were found for age, height, weight, foot size, number of years playing at the competitive level, and hours training per week, as well as scores from MABC assessment tool. Results. A series of independent sample t-tests revealed statistical differences in endurance $(\mathrm{p}<.001, \mathrm{~d}=3.57)$, and in strength tasks ( $<.001, \mathrm{~d}=1.66)$ (sit-ups, push-ups and v-ups) in favour of soccer group. However, hockey players were more agile $(\mathrm{p}<.001, \mathrm{~d}=1.26)$ and generated more power as inferred from the long jump $(\mathrm{p}<.05, \mathrm{~d}=1.1)$. Conclusion. Overall, the results showed that some domains of movement proficiency are specific to either soccer (endurance/ strength) or hockey (agility/power). These results provided coaches with information in regards to their respective teams as well as individual players' performance, and may aid in adaptations of the respective training programmes.
\end{abstract}

\section{KEY WORDS: Soccer, Hockey, Motor Performance.}

\section{INTRODUCTION}

Newell's Model of Constraints (1) has been widely implemented in motor behaviour field. In regards to motor development the model asserts that the (developmental) status of an individual depends on the relationship between individual (structural/functional), task and environmental constraints. When one of such constraints impedes the development of certain aspects of movement repertoire, they are referred to as rate limiters or controllers (2). Thus the degree of development of different aspects of motor domain may be affected by the time spent performing different physical activities, the nature of these tasks along with the environment in which they occur, in addition to individual constraints of an individual. This model can be applied to understand the developmental patterns of children who are typically or atypically developing, as well as those who represent a more advanced portion of the population, such as elite/competitive youth athletes.

Soccer and hockey represent two of the most popular sports in North America. Children and adolescents have increased involvement in these sports to the point that even at an early age they can be identified as "rep" or "AAA", terms

*. Corresponding Author:

Eryk Przysucha, Associate Professor

School of Kinesiology, Lakehead University, 955 Oliver Road, Thunder Bay, Ontario, Canada

P7B-5E1; E-mail: eprzysuc@lakeheadu.ca 
synonymous with their elite status. Despite their young age, the players engage in extensive training to enhance their technical skills, gamerelated pedigree, and also to improve their motor performance in regards to their agility, speed, endurance and strength (3). These motor-related factors are particularly important in sports such as soccer and hockey as they are "open" and continuous in nature, involve voluntary actions of the entire body to effectively change directions and withstand body contact with opponents. From the coaching perspective, the examination of these different aspects of motor performance, under different tasks demands, may aid in evaluation of the strengths and weaknesses of players, effectiveness of the training programme, as well provide measurable goals and assist in talent identification and the prediction of future performance (4).

In competitive soccer and hockey conditioning is an essential requirement for a player, regardless of the age. It has been estimated that in soccer, for example, a player engages in running about $40 \%$ of the game, $15 \%$ in sprinting, $10 \%$ in moving backwards and 5\% moving while with possession of the ball (5). Thus, soccer requires a balance of cardiovascular and muscular endurance, explosive power, and agility which all represent an important component of every-day training and predictors of successful performance (6). On the other hand, a hockey game is characterized by multiple bouts of high-intensity activity, with several recovery periods. At the youth level a player typically plays around 15 to 20 minutes of a 60-minute game, with on-ice shifts lasting between 45 to 80 seconds in duration. This performance tends to be anaerobic in nature with many changes of direction between forward and backward skating, and frequent body contact (7). To enhance the abilities of the hockey players to meet the demands of the game, at the youth level, coaches historically placed emphasis exclusively on on-ice training specific to skating, with or without the puck (e.g., power skating). However, in the last few decades more and more hockey schools or clinics started to implement off ice training known as "dry-lands", involving running, jumping, core strength exercises and static and dynamic stretches in order to improve the overall physical capabilities of the athletes, and their performance on ice.

In regards to measurement of the related fitness constructs many different field tests have been developed. Generally, these tools show good reliability as well as validity, as they correlate highly with laboratory test (8). With respect to endurance, many different tests have been devised and implemented. The multi-stage 20-m shuttle run fitness test (20mMSFT) was developed by Léger and Lambert (9), and it is a popular aerobic field test that has been used among athletes of all levels, ages, and sports including soccer (10) and hockey. Aerobic capacity increases in a linear fashion between the ages of 8 to 16 years (11), with the greatest improvements emerging between the ages of $11-$ 15 years. Many studies have reported high correlations $(0.90-0.93)$ between performance in the 20mMSFT and VO max $(12,13)$, suggesting that it is a valid predictor of overall anaerobic capacity. The emerging scoring (e.g., speed at the last stage; distance covered) also revealed evidence of good reliability and validity, and agespecific norms have been established for children between 7 and 18 years of age (14).

In comparison to endurance-related tests, fewer field tests examining the anaerobic capacity of youth athletes have been established. This is not surprising given that aerobic fitness is better defined, easier to study, and has been linked to health outcomes. However, anaerobic fitness is also very relevant to sports such as basketball, hockey, football, and soccer, because it allows players to address successfully crucial movements such as cutting, changing direction, dribbling/stick handling around an opponent or scoring a goal (15). The existing anaerobic performance tests can be broadly divided into two categories. One type examines explosive muscular power in static tasks such as jumping tests. These include tests for maximum vertical and horizontal distance, using a single- or double-leg take-off. Of these, the standing broad jump is most often used in many performance test batteries, and it requires participants to use a countermovement to jump as far as possible horizontally. The other type of anaerobic tests captures efforts in more dynamic contexts involving performance lasting up to about $10-15$ seconds. These shortduration, maximal intensity anaerobic tasks include straight line sprinting or sprint-related tests involving changes in direction, often being referred to as "agility tests". The latter kind of tests appear to be more specific to sports such as hockey and soccer, as they allow inferring 
the ability to change the direction of the body rapidly as a result of a combination of strength, speed, balance and coordination. Although there is no "gold standard" agility test, in soccer a T-test protocol, developed by Semenick (16) has been widely used (17).

Tests of muscular strength and muscular endurance have been combined into one broad fitness category because the primary consideration is determining the functional health status of the musculoskeletal system. Although generally strength is defined as the ability of a muscle or a group of muscles to produce force in one maximum resisted effort either statically or dynamically, the notion of muscular endurance is more relevant to children. Here, the emphasis is not placed on one maximum effort but rather on the ability to perform as many repetition of an exercise (e.g., push-up; sit-up) or maintaining a particular form or posture for a given time period (18). A formal assessment test which is used to measure strength/muscular endurance, among many other motor-related domains, in youth is Bruininks-Oseretsky Test of Motor Proficiency (BOM) (19). It is a reliable and valid measure used by physical and occupational therapists in the clinical setting. Also, the test provides age and gender specific norms which can be translated into population-related percentiles.

Thus far in the literature there have been numerous studies that examined the different aspects of motor performance in soccer and hockey players of different ages and skill levels. However, there is no research which compared the elite youth players across the two sports, across various related physical domains. Thus, the goal of this exploratory research was to gain an insight into potential differences in selected sports-specific domains between competitive level youth soccer and hockey players.

\section{MATERIALS AND METHODS}

Participants. Prior to commencement of data collection informed consent was obtained from each participant included in the study and the study protocol conformed to the ethical guidelines as reflected in a priori approval by the institution's human research committee. Using a purposive sampling method, two local travelling (elite) soccer and hockey teams composed of 9-year-old male players were recruited. In order to take part in the study, the players had to be one-sport athletes, be involved in their particular sport for at least 3 years, and be free of injury at the time of testing. In total, 65 participants were originally recruited, but 5 players were excluded as they were also competitive runners. As a result, 30 soccer and hockey players took part in the study. The goalies for the respective teams were tested but their data was not included in the analysis. No significant differences at the onset of the study were found in terms of their age $\left(\mathrm{M}_{\text {soccer }}=9\right.$ years, $\mathrm{SD}=3.4$ months vs. $\mathrm{M}_{\text {hockey }}=9, \mathrm{SD}=6.2$ months), hours spent training per week $\left(\mathrm{M}_{\text {soccer }}=\right.$ $5, \mathrm{SD}=1.4)$ vs. $\mathrm{M}_{\text {hockey }}=4, \mathrm{SD}=2.3$ ), weight $\left(\mathrm{M}_{\text {soccer }}=40.38 \mathrm{~kg} ; \mathrm{SD}=13.08\right.$ vs. $\mathrm{M}_{\text {hockey }}=$ $37.08 \mathrm{~kg} ; \mathrm{SD}=6.52)$, height $\left(\mathrm{M}_{\text {soccer }}=139.38 \mathrm{~cm}\right.$; $\mathrm{SD}=9.46$ vs. $\left.\mathrm{M}_{\text {hockey }}=136.42 \mathrm{~cm} ; \mathrm{SD}=4.87\right)$ and foot length $\left(\mathrm{M}_{\text {soccer }}=21.13 \mathrm{~cm}, \mathrm{SD}=1.46 \mathrm{vs}\right.$. $\left.\mathrm{M}_{\text {hockey }}=21.33 \mathrm{~cm} ; \mathrm{SD}=1.07\right)$. Also, no significant differences in MABC assessment test were found between soccer $(\mathrm{M}=4.5, \mathrm{SD}=2.24)$ and hockey $(\mathrm{M}=5.0, \mathrm{SD}=1.60)$ players indicating that developmentally their overall their movement status, as inferred across domains such as balance control, ball skills and fine-motor skills, was homogeneous.

Protocol. Prior to the commencement of the formal assessments the players attended an information session, at which point they were introduced to the tests and asked to perform practise trials. Also, at this time the participants' height $(\mathrm{cm})$ and weight $(\mathrm{kg})$ were recorded. To measure their base of support, the participant was asked to step with one foot on a piece of paper and the distance between the heel and the distal point of the largest toe was recorded $(\mathrm{cm})$. Also, a formal assessment tool, Movement Assessment Battery for Children test (20) was administered at this time individually. The scores from this test were used to infer if developmentally the players were at the same/similar status. The introductory session lasted approximately 90 minutes.

The formal testing was initiated within a week of the introductory session, and it was carried out across two sessions to prevent the impact of fatigue. Both, hockey and soccer teams were examined separately, however the players from each team were tested in groups of 3 or 4 at one time. Each athlete wore runners, a t-shirt, and shorts. Prior to each testing, a standardized 20 minute dynamic warm up was conducted. The warm up involved jogging across an artificial indoor soccer field including side shuffles, "karaoke" skips, high knees, hip flexor rotations, lunges, arm circles, and back 
rotations. After the warm up, the participants were involved in a two-station testing. The order of the tests was the same for all the participants.

On day 1 the endurance test was administered. The implementation of station 2 occurred on the subsequent day and it involved agility task, followed by station 3 which incorporated the strength tests. The participants were allowed a 20 minute recovery after the agility test prior to the initiation of the strength tasks. Once the testing was completed by all participants, a cool down was carried out.

Instruments. The first station involved the implementation of the original Léger's $20-\mathrm{m}$ shuttle run test (20mSRT) with 1-min stages, paced by beeps from the CD. At each stage, the required running speed increased until the participant could no longer reach the $20-\mathrm{m}$ distance on cue. Each stage included seven laps.
According to original protocol the athletes started at a speed of $8.5 \mathrm{~km} / \mathrm{h}$, with increments of 0.5 $\mathrm{km} / \mathrm{h}$ each minute. Due to the fact that players were aware of the test requirements from the introductory session, and to prevent fatigue, only one trial was administered. The second station involved the T-test, which examined the agility of the player. T-Test was administered using the protocol outlined by Semenick (16). The subject began the exercise with both of his feet behind starting point (see Figure 1), and after the sound signal the athlete sprinted $9.14 \mathrm{~m}$ forward, and touched the cone. Then, he shuffled $4.57 \mathrm{~m}$ to the left and touched the cone, and likewise shuffled $9.14 \mathrm{~m}$ to the right and touched the cone on the right. Consequently, the player sprinted $4.57 \mathrm{~m}$ to the left, and back to the middle cone. Then, the player ran backward passing the finish line from where he started. The time was captured by a stop-watch. Three trials were administered.

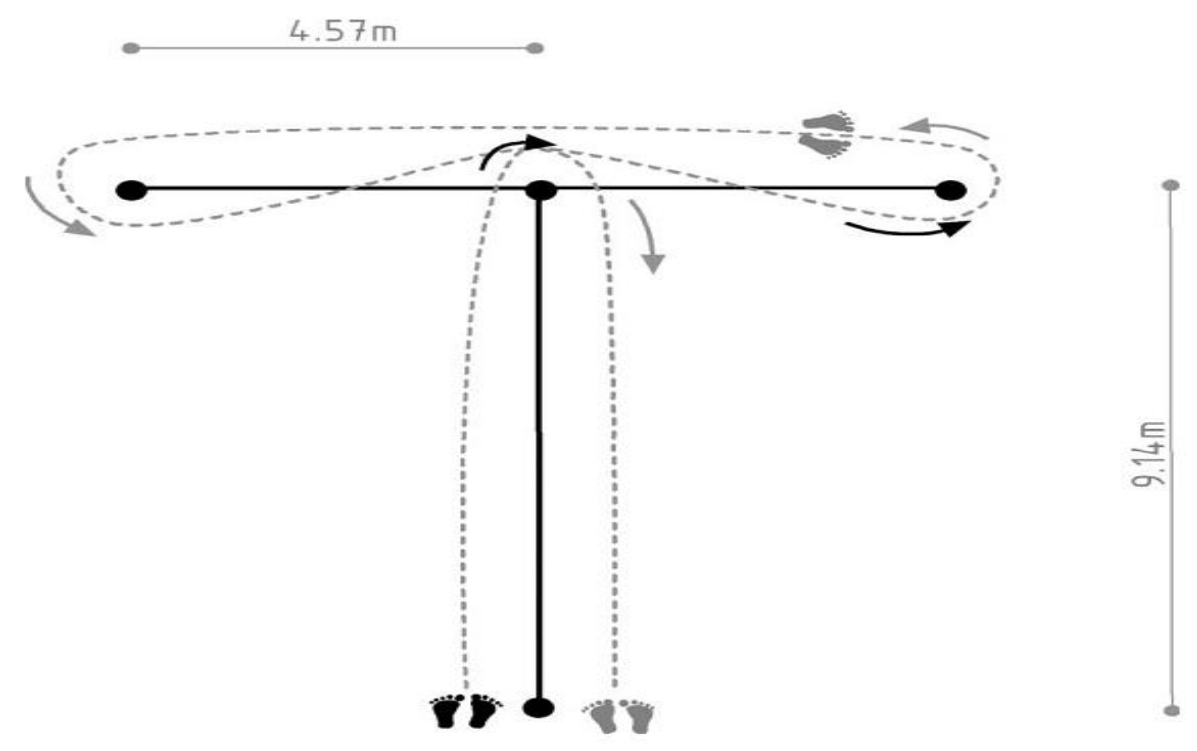

Figure 1. The lay-out of the T-Test.

The last station administered involved strength testing via push-ups, sit-ups, wall-sit, a v-up task, and a long jump. The items were derived from the Bruinink's assessment test (BOT-2) (19). This is a norm referenced test which examines fine and gross motor skills of children and youth, 4 through 21 years of age. For this study, only the strength section was implemented. In regards to the push-ups and sit-ups, the participant was asked to do as many repetitions as he could in 30 seconds. The v-up and wall-sit were completed with proper form to the maximum of 1 minute (see Figure 2). The v-up only counted if the participant maintained his legs and arms straight and off the ground. The wall-sit trial only counted if the participant kept his knees flexed at 90 degrees, with heels in contact with the ground, and arms crossed, while the upper body was in contact with the wall. The last task involved a two-foot horizontal jump, with arms being used for a counter-movement motion. The distance 
between the starting line and the heel of the foot closest to the line was used to infer the degree of performance. The periods between each exercise were used for demonstration and rest.

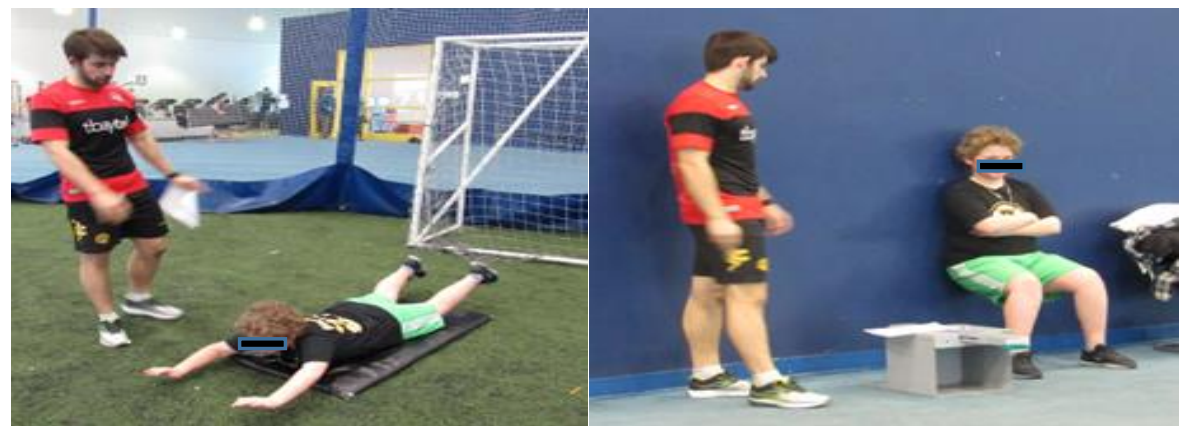

Figure 2. Illustration of the V-up (on the left) and the wall-sit tasks.

Data Analysis. In order to examine the differences between the two groups a series of independent samples t-tests were implemented, at alpha $\mathrm{p}<.05$. Also the effect sizes were calculated in the form of d statistic (21). For each analysis the assumptions for normality (ShapiroWilk test) and homogeneity of variance (Levene's test) were also verified.

The endurance was inferred from two variables, namely the speed at the last completed stage $(\mathrm{km} / \mathrm{h})$, and the number of laps completed. In the case of the former, the lower value indicated better performance, whereas higher number of laps completed coincided with better outcome. The agility was examined via time to completion of the T-test, expressed in seconds, with smaller values indicating a more positive outcome. The average of three trials was used for the analysis. Finally, the strength was inferred from the composite score resulting from the four previously described items. As per test guidelines, only one trial was administered for all the tasks. In order to make inferences in regards to the normative data, the scores were combined into a Total Point Score, with a maximum value of 42 . Subsequently, this score was transformed into a scale score, which was used for the purpose of the analysis and it allowed to make inferences in regards to the corresponding percentiles scores. The higher scores indicated a more proficient performance, in relation to the age/gender specific norms.

\section{RESULTS}

In regards to endurance, the analysis of the speed at the final stage revealed that the soccer players performed better $(\mathrm{M}=12.63 ; \mathrm{SD}=.49)$ than the hockey group $(\mathrm{M}=9.13 ; \mathrm{SD}=.86)(\mathrm{t}$ $(58)=13.82, p<.001, \mathrm{~d}=3.57)$. The same scenario emerged when the number of laps completed was examined as once again soccer group out performed $(\mathrm{M}=46.70 ; \mathrm{SD}=4.99)$ the hockey players $(\mathrm{M}=36.16 ; \mathrm{SD}=9.89)(\mathrm{t}(58)=$ $5.49, p<.001, \mathrm{~d}=1.46)$. The analysis of strength, as inferred from the composite percentile score, also showed that soccer group placed higher $(\mathrm{M}=$ 82.34 , $\mathrm{SD}=7.07$ ) as compared to the hockey players $(\mathrm{M}=70.46, \mathrm{SD}=9.79)(\mathrm{t}(58)=6.43, p<$ $.001, \mathrm{~d}=1.66)$. In regards to the individual subtasks, composing the strength section of Bruiniks, soccer players achieved higher scores in regards to push-ups $(\mathrm{M}=18.4$ vs. $\mathrm{M}=12.2)$, sit-ups ( $\mathrm{M}$ $=38.3$ vs. $\mathrm{M}=26.4)$, wall-sit $(\mathrm{M}=52.4 \mathrm{sec}$ vs. $\mathrm{M}$ $=39.2)$ and v-ups $(\mathrm{M}=60.0 \mathrm{sec}$ vs $\mathrm{M}=49.6)$. However, this was not the case in regards to standing long jump where the hockey players exhibited substantially better performance $(\mathrm{M}=$ 68.3 inches vs. $\mathrm{M}=51.6)(\mathrm{t}(58), p<.05, \mathrm{~d}=1.1)$. The analysis of the agility, as inferred from the TTest, also showed that the hockey players completed the course faster $(\mathrm{M}=12.20 ; \mathrm{SD}=$ 1.32) as compared to the soccer group ( $\mathrm{M}=$ 15.70; $\mathrm{SD}=3.67)(\mathrm{t}(58)=4.91, p<.001, \mathrm{~d}=$ 1.26).

\section{DISCUSSION}

Cardiorespiratory fitness is the main health related factor across general population as well as in many sports settings. One of the most widely used field tests for estimating cardiorespiratory fitness is the 20-m shuttle run test (9). The 20-m 
shuttle run test has been shown to be a reliable and valid method of estimating and predicting $\mathrm{VO}_{2}$ max in children and adolescence. Also, from the ecological validity standpoint, it applies well to sports where aerobic fitness helps to sustain a high work rate and assist in rapid recovery between short, intermittent bouts of highintensity efforts. The results of the present study showed that across both variables (speed at last stage; number of laps) the soccer players outperformed their hockey counterparts. The results from the inferential statistics were confirmed by large effect sizes (d statistic), further supporting the fact that between groups differences were meaningful. Also, as evident from relatively small degree of intra-group variability, as captured by group standard deviations, the performance was relatively stable among the respective groups indicating that this "trend" was consistent among all the participants, within the same group. Given that the two groups did not differ in regards to the key morphological characteristics, it could be concluded that the type of sport, and respective training, resulted in the emerging differences. These findings are not surprising. The game of soccer depends heavily on the aerobic endurance as the training involves technical and functional drills (e.g., small-sided games) which require bouts of prolonged running at maximum or sub maximum intensities, with some walking or light jogging in-between. The same scenario applies to the actual games, where given the size of the pitch and the amount of space to cover, the players are involved in continuous movement with frequent bouts of sprints and recovery-runs. Also, in line with the characteristics/rules of soccer the player remains "in the game" for prolonged periods of time with the necessity to recover in-between sprints while walking or jogging. On the other hand, in hockey, the players are generally involved in 45 to 90 seconds shifts, and their recovery is more static as they are off the ice for several minutes before returning to active play. The practises also more often than not resemble the game-like situations where the players engage in skating for a short period of time, with frequent breaks. Hence, due to the constraints of the sport, the hockey players are generally not forced to rely on cardiovascular endurance as much as the soccer players.

In regards to strength, the soccer players also performed better than the hockey group, as inferred from the composite score for the respective subsection of the Bruiniks test (19). However, as evident from the individual 4 subscores, the nature of these differences emerged across push-ups and sit-ups, as well as tasks which focused on the overall core strength, but not in the long jump where the descriptive statistics showed substantial differences in favour of hockey players. While strength and power are often used interchangeably, they represent two different concepts. Hence, the fact that the groups performed differently across both domains is not surprising. Generally, horizontal jumping task is considered to capture anaerobic power, and it correlates highly with skating speed in professional players (22). Having a high amount of muscular power means that a heavy load can be lifted or moved quickly, such as the explosive movement of pushing off and starting the skating motion quickly, accelerating and changing skating speed (23). Hence, anaerobic fitness, more specifically power, is important to hockey players because of the many rapid spurts of energy that are involved (24). The present results seem to confirm this general trend.

The last construct that was assessed was agility, as inferred from the scores on the T-test. Agility can be defined in many ways, however here it is viewed as the ability to change direction rapidly, without losing balance, using power and neuromuscular coordination. Since in soccer, agility constitutes around $11 \%$ of player movement, and on average a soccer player changes direction every 2-4 seconds (17), it was expected that soccer players would be superior to the hockey players. However, the data failed to confirm this hypothesis. Considering that all the factors which contribute to the development of the agility (e.g., chronological age, somatic maturation, body size and estimated leg/foot length), were constant across the groups, the differences between the groups have to be attributed to the training implemented. Research has shown that muscle activation during running and skating is drastically different, and that the neuromuscular demands of skating have more pronounced effects on power (25). This is evident from the activation of various muscles (e.g., biceps femoris, gluteus maximus, vastus medialis, gastrocnemius and tibialis anterior), which are involved in stopping, re-starting and transition between forward, backward, diagonal 
and lateral movements (24). From the standpoint of training, it should also be pointed out that these aspects of skating are very technical, hence a substantial amount of time is spent during the practises to improve its mechanics. This is particularly true for youth athletes. Also, the fact that the area of contact with the surface is so much smaller in hockey, as compared to soccer, this skill requires a more refined recruitment of muscle groups which not only result in more power but also in better balance to prevent falling or stumbling.

\section{CONCLUSION}

Even though both games require a combination of anaerobic and aerobic fitness in order to endure the long periods of physical activity with frequent bursts of power, soccer seems to be more anaerobic whereas hockey is more anaerobic, at least among the youth athletes. Hence, the present results do not indicate that one group is more physically fit than the other, but rather that even at this early age the two sports generate different kind of athletes. It appears that elite performance in hockey, at this developmental stage, is not as dependent on strength and endurance, as is on speed, agility, and power. The reverse appears to be true for youth elite soccer players. In regards to the potential limitations, the external validity of the emerging inferences should be treated with caution due to relatively small sample size. Nevertheless, the results do suggest that different aspects of training should be emphasized in both groups to enhance the "limiting" factors in their performance as they further develop in their athletic careers (1).

\section{APPLICABLE REMARKS}

- Assessment of motor abilities among even young (elite) athletes provides insight into their strengths and possible areas for improvement.

- The fact that different sports affect the improvement of various aspects of motor performance should encourage parents, coaches as well as teachers to allow young (gifted) athletes to take part in many different sports, particularly early on during their physical development.

\section{REFERENCES}

1. Newell K. Constraints on the development of coordination. In M.G. Wade \& H.T.A. Whiting (Eds) (1986), Motor development in children: Aspects of coordination and control (pp. 341-361). Amsterdam: Niijhoff. [DOI:10.1007/978-94-009-4460-2_19]

2. Thelen E. Motor development: A new synthesis. American Psychologist. 1995; 50: 79-95. [DOI:10.1037/0003066X.50.2.79] [PMID]

3. Ransdell LB, Murray T. A physical profile of elite female ice hockey players from the United States. Journal of Strength and Conditioning Research. 2011: 25(9): 2358-2363. [DOI:10.1519/JSC.0b013e31822a5440] [PMID]

4. Armstrong N, Barker AR. Endurance training and elite young athletes. Medicine and Sport Science. 2010; 56: 5983. [DOI:10.1159/000320633] [PMID]

5. Reilly T. Physiological aspects of soccer. Biology and Sport. 1994; 11: $3-20$.

6. Buchheit M, Delhomel G, Ahmaidi S. Time-motion analysis of elite young French soccer players. Coaching Sport Science. 2008; 3: $21-26$.

7. Roczniok R, Stanula A, Maszczyk A, Mostowik A, Kowalczyk M, Fidos-Czuba O, Zając A. Physiological, physical and on-ice performance criteria for selection of elite ice hockey teams. Biology of Sport. 2016; 33: 43-48. [DOI:10.5604/20831862.1180175] [PMID] [PMCID]

8. Bosquet L, Léger L, Legros P. Methods to determine aerobic endurance. Sports Medicine. 2002; 32(11): 675-700. [DOI:10.2165/00007256-200232110-00002] [PMID]

9. Leger L, Lambert J. A maximal 20-m shuttle run test to predict V02 max. European Journal of Applied Physiology. 1982: 49: 1-12. [DOI:10.1007/BF00428958]

10. Caldwell BP, Peters DM. Seasonal variation in physiological fitness of a semi-professional soccer team. Journal of Strength and Conditioning Research. 2009; 23(5): 1370-1377. [DOI:10.1519/JSC.0b013e3181a4e82f] [PMID]

11. Roemmich JN, Rogol AD. Physiology of growth and development. Its relationship to performance in the young athlete. Clinical Sports Medicine. 1995; 14(3): 483-502. [PMID] 
12. Paliczka VJ, Nichols AK, Boreham CA. A multistage shuttle run as a predictor of running performance and maximal oxygen uptake in adults. British Journal of Sports Medicine. 1987; 21 (4): 163-164. [DOI:10.1136/bjsm.21.4.163] [PMID] [PMCID]

13. Ramsbottom R, Brewer J, Williams C. An examination of the maximal multistage shuttle run test as a predictor of V02 max in active female subjects. Journal of Sports Sciences. 1988; 6: 165A.

14. Tomkinson GR, Lang JJ, Tremblay MS, Dale M, LeBlanc AG, Belanger K, Ortega F, Léger L. International normative $20 \mathrm{~m}$ shuttle run values from 1142026 children and youth representing 50 countries. British Journal of Sports Medicine. 2016; 1: 1-14.

15. Hammami R, Chaouachi A, Makhlouf I, Granacher U, Behm DG. (2016). Associations between balance and muscle strength, power performance in male youth athletes of different maturity status. Pediatric Exercise Science. 2016; 28 (4), 2-16. [DOI:10.1123/pes.2015-0231]

16. Semenick D. Test and measurements: The T- test. National Strength and Conditioning Association Journal. 1990; 12(1): 36-37. https://doi.org/10.1519/0744-0049(1990)012<0036:TTT>2.3.CO;2 [DOI:10.1519/07440049(1990)0122.3.CO;2]

17. Sporis G, Jukic I, Milanovic L, Vucetic V. Reliability and factorial validity of agility tests for soccer players. Journal of Strength and Conditioning Research. 2010; 24(3): 679-686. [DOI:10.1519/JSC.0b013e3181c4d324] [PMID]

18. Meckel Y, Machnai O, Eliakim A. Relationship among repeated sprint tests, aerobic fitness, and anaerobic fitness in elite adolescent soccer players. Journal of Strength and Conditioning Research. 2009; 23(1): 163-169. [DOI:10.1519/JSC.0b013e31818b9651] [PMID]

19. Bruininks RH, Bruininks BD (BOT-2). Bruininks-Oseretsky Test of Motor Proficiency Second Edition. 2005; Minneapolis, Minnesota: Pearson Assessments.

20. Henderson SE, Sugden DA, Barnett AL. Movement Assessment Battery for Children - 2. 2007; London: Harcourt Assessment.

21. Cohen J. Statistical power analysis for the behavioral sciences (2nd ed.). 1998; Hillsdale, NJ: Lawrence Earlbaum Associates.

22. Mascaro T, Seaver BL, Swanson L. Prediction of skating speed with off-ice testing in professional hockey players. Journal of Orthopedics and Sports Physical Therapy. 1992;10: 92-98. [DOI:10.2519/jospt.1992.15.2.92] [PMID]

23. Behm DG, Wahl MJ, Button DC, Power KE, Anderson KG. Relationship between hockey skating speed and selected performance measures. The Journal of Strength \& Conditioning Research. 2005; 19(2): 326-331. https://doi.org/10.1519/R-14043.1 [DOI:10.1519/00124278-200505000-00015] [PMID]

24. Bracko M. On-ice performance characteristics of elite and non-elite women's ice hockey players. Journal of Strength and Conditioning Research. 2001; 15(1): 42-47. [DOI:10.1519/00124278-200102000-00020] [PMID]

25. Pearsall DJ, Turcott RA, Murphy SD. Biomechanics of Ice Hockey. Exercise and Sport Science. $2000 ; 23: 32-39$. 